\title{
No evidence for enhanced processing of speech that is low-pass filtered near the edge frequency of cochlear dead regions in children.
}

DOI:

10.1080/14992027.2018.1460496

\section{Document Version}

Accepted author manuscript

Link to publication record in Manchester Research Explorer

Citation for published version (APA):

Malicka, A., Wilson, W., Baer, T., Munro, K., Baker, R., Miluzzi, D., \& Moore, B. (2018). No evidence for enhanced processing of speech that is low-pass filtered near the edge frequency of cochlear dead regions in children. International Journal of Audiology. https://doi.org/10.1080/14992027.2018.1460496

\section{Published in:}

International Journal of Audiology

\section{Citing this paper}

Please note that where the full-text provided on Manchester Research Explorer is the Author Accepted Manuscript or Proof version this may differ from the final Published version. If citing, it is advised that you check and use the publisher's definitive version.

\section{General rights}

Copyright and moral rights for the publications made accessible in the Research Explorer are retained by the authors and/or other copyright owners and it is a condition of accessing publications that users recognise and abide by the legal requirements associated with these rights.

\section{Takedown policy}

If you believe that this document breaches copyright please refer to the University of Manchester's Takedown Procedures [http://man.ac.uk/04Y6Bo] or contact uml.scholarlycommunications@manchester.ac.uk providing relevant details, so we can investigate your claim.

\section{OPEN ACCESS}




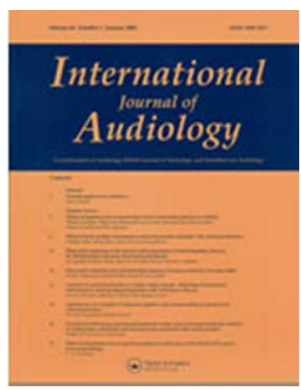

\section{No evidence for enhanced processing of speech that is low- pass filtered near the edge frequency of cochlear dead regions in children}

\begin{tabular}{|r|l|}
\hline Journal: & International Journal of Audiology \\
\hline Manuscript ID & TIJA-2017-09-0308.R2 \\
\hline Manuscript Type: & Original Paper \\
\hline Date Submitted by the Author: & n/a \\
\hline Complete List of Authors: & $\begin{array}{l}\text { Malicka, Alicja; La Trobe University College of Science Health and } \\
\text { Engineering, School of Allied Health } \\
\text { Wilson, Wayne; The University of Queensland, School of Health and } \\
\text { Rehabilitation Sciences; } \\
\text { Baer, Thomas; University of Cambridge, Department of Experimental } \\
\text { Psychology } \\
\text { Munro, Kevin; University of Manchester, School of Psychological Sciences } \\
\text { Baker, Richard; The University of Manchester, School of Psychological } \\
\text { Sciences } \\
\text { Miluzzi, Deanna; The University of Queensland, School of Health and } \\
\text { Rehabilitation Sciences } \\
\text { Moore, Brian; University of Cambridge, Dept of Experimental Psychology }\end{array}$ \\
\hline Keywords: & $\begin{array}{l}\text { Speech Perception, Psychoacoustics/Hearing Science, Behavioral Measures, } \\
\text { Conditions/Pathology/Disorders }\end{array}$ \\
\hline
\end{tabular}


No evidence for enhanced processing of speech that is low-pass filtered near the edge frequency of cochlear dead regions in children

\author{
Alicja N. Malicka, ${ }^{1,2}$ Wayne J. Wilson, ${ }^{2}$ Thomas Baer, ${ }^{3}$ Kevin J. Munro, ${ }^{4}$ Richard J. Baker, ${ }^{4}$ \\ Deanna Miluzzi², and Brian C.J. Moore ${ }^{3}$ \\ ${ }^{1}$ School of Allied Health, La Trobe University, Australia \\ ${ }^{2}$ School of Health and Rehabilitation Sciences, The University of Queensland, Australia \\ ${ }^{3}$ Department of Experimental Psychology, University of Cambridge, UK \\ ${ }^{4}$ Manchester Centre for Audiology and Deafness, School of Health Sciences, University of \\ Manchester, UK
}

\title{
Key Words
}

Cochlear dead regions, speech perception, neuroplasticity, low-pass filtered speech

\author{
Abbreviations \\ $\mathrm{DR}=$ Cochlear dead region \\ $\mathrm{f}_{\text {edge }}=$ Edge frequency of DR \\ SII $=$ Speech intelligibility index \\ $\mathrm{VCV}=$ Vowel Consonant Vowel nonsense syllable
}

\section{Address for correspondence}

Alicja Malicka

School of Allied Health, La Trobe University

Bundoora, VIC 3083, Australia

Email: a.malicka@,latrobe.edu.au 


\section{Key Words}

Cochlear dead regions

Speech perception

Neuroplasticity

Low-pass filtered speech 
No evidence for enhanced processing of speech that is low-pass filtered near the edge frequency of cochlear dead regions in children

\author{
Alicja N. Malicka, ${ }^{1,2}$ Wayne J. Wilson, ${ }^{2}$ Thomas Baer, ${ }^{3}$ Kevin J. Munro, ${ }^{4}$ Richard J. Baker, ${ }^{4}$ \\ Deanna Miluzzi², and Brian C.J. Moore ${ }^{3}$ \\ ${ }^{1}$ School of Allied Health, La Trobe University, Australia \\ ${ }^{2}$ School of Health and Rehabilitation Sciences, The University of Queensland, Australia \\ ${ }^{3}$ Department of Experimental Psychology, University of Cambridge, UK \\ ${ }^{4}$ Manchester Centre for Audiology and Deafness, School of Health Sciences, University of \\ Manchester, UK
}

\title{
Key Words
}

Cochlear dead regions, speech perception, neuroplasticity, low-pass filtered speech

\author{
Abbreviations \\ $\mathrm{DR}=$ Cochlear dead region \\ $\mathrm{f}_{\text {edge }}=$ Edge frequency of DR \\ SII $=$ Speech intelligibility index \\ $\mathrm{VCV}=$ Vowel Consonant Vowel nonsense syllable
}

\section{Address for correspondence}

Alicja Malicka

School of Allied Health, La Trobe University

Bundoora, VIC 3083, Australia

Email: a.malicka@,latrobe.edu.au 


\begin{abstract}
Objectives: Cochlear dead regions (DRs) are regions in the cochlea where the inner hair cells and/or neurons are not functioning. Adults with extensive high-frequency DRs have enhanced abilities in processing sounds with frequencies just below the edge frequency, $\mathrm{f}_{\text {edge, }}$, of the DR. It was assessed whether the same is true for children. Design: Performance was compared for children aged 8 to 13 years with: DRs (group DR), hearing impairment but without DRs (group NODR), and normal hearing (group NH). Seven ears in each group were tested. Each ear in the DR group was matched in age and low-frequency hearing with an ear in the NODR group, and in age with an ear in the NH group, giving seven "triplets". Within each triplet, the percent correct identification of vowel-consonant-vowel stimuli was measured using stimuli that were low-pass filtered at $f_{\text {edge }}$ and $0.67 f_{\text {edge, }}$, based on the ear with a DR. For the hearing-impaired ears, stimuli were given frequency-selective amplification as prescribed by DSL 4.1. Results: No significant differences in performance were found between groups for either low-pass cut-off frequency. Conclusion: Unlike adults, the children with DRs did not show enhanced discrimination of speech stimuli with frequencies below $f_{\text {edge. }}$
\end{abstract}


Neuroplasticity can be defined as the ability of the nervous system to reorganize its structure, function and connections in response to intrinsic and extrinsic factors (Kral et al., 2001). It can occur during normal development or as a result of disease or therapy. Neuroplasticity can result in improved function, sometimes called adaptive plasticity, or in impaired function, sometimes called maladaptive plasticity (Cohen et al., 1997; Nudo, 2006). For example, the improvement in speech identification performance over the first few months following fitting of a cochlear implant may reflect adaptive plasticity, whereas tinnitus may reflect maladaptive plasticity in the central auditory system's response to altered peripheral input (Eggermont, 2017).

It is well known that sensory deprivation can result in reorganization of cortical areas associated with the deprived modality (for a review, see Irvine \& Wright, 2005). Animal studies have shown that damage to a restricted region of the basilar membrane can cause the part of the auditory cortex responsible for processing information from that region to become tuned to the input from an adjacent, undamaged region (Robertson \& Irvine, 1989). Moreover, the level and the extent of the reorganization depend strongly on when the peripheral lesion was induced during development, with greater effects expected for lesions induced early in life (Harrison, 2001; Kral et al., 2001).

In this study we investigated possible functional changes associated with dead regions (DRs) in the middle and basal regions of the cochlea, which are tuned to medium and high frequencies, for the processing of low-pass filtered speech. DRs are regions in the cochlea where the inner hair cells, synapses, and/or neurons are not functioning. If a DR starts at a medium or high frequency and extends to the basal end of the cochlea, it is called a continuous DR and it is entirely characterized by its edge frequency ( $\left.f_{\text {edge }}\right)$, which corresponds to the characteristic frequency of the inner hair cells and neurons immediately adjacent to the DR (Moore, 2001; Moore \& Vinay, 2009). The effect of a continuous basal 
DR on the processing of a sinusoid in the cochlea depends on the value of $f_{\text {edge }}$ and on the frequency and intensity of the sinusoid. For a low-intensity sinusoid with a frequency above $\mathrm{f}_{\text {edge, }}$, the peak of the basilar-membrane vibration pattern will fall within the DR and the vibration will not be detectable. If the intensity of the sound is increased sufficiently, the larger basilar-membrane vibration within the DR will still not be detected, but the basilarmembrane vibration outside the DR may become large enough to stimulate neurons tuned to frequencies just below $\mathrm{f}_{\text {edge }}$ and this may allow the sound to be detected (Moore, 2001; Moore, 2004; Moore \& Malicka, 2013). Detection of a sound in this way is called "off-place" or "off-frequency" listening.

It has been proposed that for adults with acquired basal DRs, cortical neurons that formerly responded to frequencies within the DR will eventually become responsive to frequencies just below $f_{\text {edge. }}$ This process is referred to as "remapping" and it is thought to represent a form of neuroplasticity (Kluk \& Moore, 2006; Moore \& Vinay, 2009). The remapping of cortical neurons may result in an over-representation of frequencies just below $\mathrm{f}_{\text {edge }}$ in the auditory brainstem and cortex (Dong et al., 2010). This in turn can result in better auditory processing of those frequencies, for example enhanced frequency discrimination of sinusoids with frequencies just below $\mathrm{f}_{\text {edge }}$ (Kluk \& Moore, 2006; Thai-Van et al., 2007; Moore \& Vinay, 2009; Heggdal et al., 2013), and improved discrimination of speech stimuli that are low-pass filtered with a cut-off frequency close to $f_{\text {edge }}$ (Vestergaard, 2003; Moore \& Vinay, 2009).

While such benefits have been observed for adults with DRs, we are not aware of any studies investigating whether they occur for children with DRs. The aim of this study was to determine if children with hearing loss at medium and high frequencies and with DRs (the DR group) would show an enhanced ability to process nonsense syllables that were low-pass filtered with a cut-off frequency at or somewhat below the value of $\mathrm{f}_{\text {edge }}$ for each child, 
compared to children with comparable low-frequency hearing but without DRs (the NODR group). An age-matched group of children with normal hearing (the $\mathrm{NH}$ group) was also tested. It was hypothesized that when the stimuli included all frequencies up to $f_{\text {edge }}$ (condition 1) the DR group would outperform the NODR group, but when the stimuli included frequencies only up to $0.67 \mathrm{f}_{\text {edge }}$ (condition 2 ) the difference between the DR and NODR groups would be smaller or absent, since the enhancement effects found for adults appears to occur mainly for frequencies just below $f_{\text {edge. }}$ The cut-off frequency of $0.67 f_{\text {edge }}$ was based on studies showing enhanced frequency discrimination for frequencies from about $0.6 f_{\text {edge }}$ to $0.9 f_{\text {edge, }}$, but not for frequencies below $0.6 f_{\text {edge }}$ (Kluk \& Moore, 2006; Thai-Van et al., 2007; Moore \& Vinay, 2009; Heggdal et al., 2013). No definite hypothesis was made about the relative performance of the DR group and the NH group in conditions 1 and 2, since any beneficial effects of enhanced central processing for the DR group might be offset by suprathreshold deficits in auditory processing associated with their hearing loss for frequencies below $\mathrm{f}_{\text {edge }}$ (Moore, 2007).

\section{Method \\ Participants}

Fifteen children (nine male and six female) aged 8 to 13 years were tested. Seven children with normal hearing were sampled from the population of children of staff working at the University of Queensland. The data for these seven were newly gathered for this study. The children with sensorineural hearing loss (SNHL) were selected from the clinical records of three sources in the UK: the Audiology Department at Queen's Medical Centre in Nottingham, the Manchester Inclusion Service for Children with Hearing Needs, and Mary Hare Grammar School in Newbury. Most of the children had hearing loss from early on in their life and for most the cause of the hearing loss was unknown. Some of the data for these children were published in Malicka et al. (2013). The hearing-impaired children all wore 
behind-the-ear hearing aids. None of the hearing aids used frequency compression. Fourteen ears from these children were tested (both ears from six children and one ear each from two children, giving seven right and seven left ears). Seven ears had DRs (the DR group) and seven ears had no DRs (the NODR group). For three ears in the DR group, the DRs were restricted, having both lower and upper edge frequencies. For the other four ears in the DR group, the DRs appeared to extend from the edge frequency to the base of the cochlea (i.e. they were continuous). In what follows, $f_{\text {edge }}$ refers to the lower edge frequency of any DR. For each ear with a DR, an ear without a DR was selected so as to match the audiometric thresholds for frequencies just below $f_{\text {edge, }}$ although it was difficult to achieve perfect matches and differences in audiometric threshold up to $15 \mathrm{~dB}$, and in one case $20 \mathrm{~dB}$, did occur (see Table 1). This resulted in seven pairs of ears. Participants within each pair differed in age by no more than two years.

The NH children had hearing thresholds within normal limits (defined as audiometric air-conduction thresholds of $20 \mathrm{~dB}$ HL or better for both ears for audiometric frequencies from 0.25 to $8 \mathrm{kHz}$ ), with no conductive overlay, and type A tympanograms. One ear from each of these participants (four right ears and three left ears) was included (the NH group). Each participant was selected to match the age of one of the DR-NODR pairs. This resulted in seven triplets. Each triplet contained one ear from the DR group, its matched ear from the NODR group, and an age-matched ear from the NH group. Table 1 shows the characteristics of all test ears. Note that triplets 5 and 6 each contained DR and NODR ears from a single child (each child having bilateral SNHL but a unilateral DR). Neural plasticity can be different for children with unilateral versus bilateral DRs (see discussion).

Ethical approval was obtained from the Nottingham Research Ethics Committee 1 (REC reference number 05/Q2403/146), the University of Manchester, the School of Psychological Sciences Research Ethics Committee (Project reference number 314/05) and The University of Queensland Human Research Ethics Committee (Approval number 2012000579). Written consent was obtained from both parents and participating children. 


\section{Diagnosis of DRs}

DRs were diagnosed and the values of $\mathrm{f}_{\text {edge }}$ were estimated using the TEN(HL) test (Moore et al., 2004) and fast psychophysical tuning curves (Sek et al., 2005; Malicka et al., 2009); the results of the two tests were consistent for all children. For full details, see Malicka et al. (2013). The frequencies at which DRs were present are indicated by asterisks in Table 1 . The value of $f_{\text {edge }}$ was taken as the lowest frequency at which a DR was present.

\section{Speech stimuli}

Vowel-consonant-vowel (VCV) nonsense syllables were used to reduce the influence of lexical context and knowledge on the results. There were 12 lists of 63 syllables, each with one of 21 consonants (/p, t, k, b, d, g, f, $\left.\theta, \mathrm{s}, \int, \mathrm{h}, \mathrm{v}, \mathrm{z}, \mathrm{r}, \mathrm{l}, \mathrm{j}, \mathrm{w}, \mathrm{t} \int \mathrm{d}, \mathrm{n}, \mathrm{m} /\right)$ in the context of one of three vowels (/a, I, u/). The recorded nonsense syllables were spoken by a female speaker with a British accent.

Each combination of vowel and consonant was presented once in each list in randomized order. The speech stimuli had a nominal level of $65 \mathrm{~dB}$ SPL, before any filtering was applied. Three types of filtering were applied, although in practice these were implemented as a single combined filter for each ear. The types of filtering were: (1) compensation for the frequency characteristic of the TDH 39 headphones used, so as to give a diffuse-field response at the eardrum; (2) frequency-selective amplification as prescribed by DSL version 4.1 for speech with an overall level of 65 dB SPL (Seewald et al., 2005) only for the DR and NODR groups; and (3) low-pass filtering, as described in the next section.

\section{Low-pass Filtering}

The amplified stimuli were presented either in a broadband condition (upper cut-off 
frequency $7.5 \mathrm{kHz}$ ) or low-pass filtered with various cut-off frequencies. All low-pass filters were $250^{\text {th }}$ order finite impulse response filters, designed with the "firl" function in Matlab. The nominal cut-off frequencies are specified at the 6-dB down points. The relative responses of the low-pass filters were close to $0 \mathrm{~dB} 100 \mathrm{~Hz}$ below the cut-off frequency and about 60 dB down $110 \mathrm{~Hz}$ above the cut-off frequency. For the purpose of the current study, the results were analyzed for only two low-pass frequencies for each ear from the DR group. In condition 1 , the speech was low-pass filtered with a cut-off frequency equal to $f_{\text {edge. }}$ In condition 2 , the cut-off frequency was equal to $0.67 f_{\text {edge. }}$. For the other two ears within each triplet, the stimuli were low-pass filtered at the same frequency as for the ear in that triplet with a DR. When the matched NODR and NH ears in a triplet had not been tested using the selected cut-off frequency, the score for the selected cut-off frequency was estimated by linear interpolation (on a logarithmic frequency scale) from scores for adjacent frequencies, or in one case by extrapolation.

\section{Calculation of the Speech Intelligibility Index (SII)}

The speech intelligibility index (ANSI, 1997) can be used to predict the intelligibility of speech based largely on the audibility of different parts of the speech spectrum. SII values were calculated for each ear and condition for two reasons: (1) To ensure that performance predicted on the basis of audibility would be similar for DR ears and their matched NODR ears, so that any difference in performance could not be attributed to differences in audibility; (2) To allow performance to be compared with that expected based on audibility. SII values were calculated using the one-third-octave-band method and the frequency-importance weighting function specified in ANSI S3.5-1997 (1997) for nonsense syllables and a nonreverberant environment. The transfer function relating predicted intelligibility to the SII value was that derived by Malicka et al. (2013). 


\section{Equipment}

The prerecorded stimuli were replayed using a PC equipped with an external 16-bit sound card (Edirol UA-5), using a sampling rate of $16 \mathrm{kHz}$. The output of the sound card was fed to the external input channel of a Kamplex KC35 clinical audiometer, which was used to control the overall level. The participants listened to the speech monaurally via TDH 39 headphones (with MX41/AR cushions) connected to the audiometer. The system was calibrated as described by Malicka et al. (2013).

\section{Procedure}

Children were tested in a sound-attenuating room over 2-3 sessions, each lasting up to two hours (including breaks). During session one, the type and the degree of hearing loss were established and the presence/extent of DRs, if any, was determined. During the remaining sessions the speech tests were administered. The child was first asked to read aloud the 21 consonants used in the VCV nonsense syllables, which were presented on the computer screen. Next, one training list was presented. The child was asked to indicate which consonant they heard by clicking on that consonant from a visual depiction of all consonants presented on the computer screen. Finally, the experimental conditions were presented. Three lists were selected randomly from the 12 lists of 63 syllables for testing each low-pass filter condition; no list was repeated. The percentage of correctly identified consonants across the three lists was calculated for each test ear.

\section{Data analysis}

In addition to percent correct scores, we calculated scores relative to what would be expected on the basis of audibility, by dividing the mean score for each ear for each low-pass filter 
condition by the score predicted from the SII for that ear and that condition. These are referred to as "SII-relative" scores. Ratios greater than 1 indicate performance better than expected based on audibility, while ratios less than 1 indicate performance worse than expected based on audibility.

Non-matched samples t-tests (two-tailed) were used to determine if mean scores or ratio scores differed between the DR and NODR groups, the DR and NH groups, and the NODR and NH groups. A significance level of $0.05 / 3$ was used to allow for multiple comparisons.

\section{Results}

Condition 1 - low-pass cut-off frequency equal to $f_{\text {edge }}$

Figure 1 shows, for each test ear in each triplet, the mean score across the three lists when the low-pass filter had a cut-off frequency equal to $\mathrm{f}_{\text {edge }}$ (open symbols). The scores predicted from the SII (filled symbols) were similar for the ears within each triplet, confirming that audibility was well matched across groups. The ears with DRs did not generally perform better than the ears without DRs or than the NH ears. The t-tests showed that the scores did not differ significantly (at the adjusted $p$ value of 0.0167 ) for the DR versus NODR groups ( $t$ $=-1.806, p=0.12)$, the DR versus NH groups $(t=-3.015, p=0.02$; note that the mean score was lower for the DR group than for the NH group), or the NODR versus NH groups ( $t=$ $-1.307, p=0.24)$.

Figure 2 shows, for each test ear in each triplet, the SII-relative scores when the lowpass filter had a cut-off frequency equal to $\mathrm{f}_{\text {edge. }}$ If DRs led to neuroplasticity that enhanced the processing of sounds with frequencies just below $f_{\text {edge, }}$, then ears with DRs should show SII relative scores above 1 . In fact the ratios were slightly below 1 (in the range 0.67 to 0.86 ) for five of the triplets and slightly above 1 (1.04 and 1.16) for triplets 5 and 6 . Also, the SII 
relative scores were not consistently higher for the DR group than for the NODR or NH groups. The t-tests showed no significant differences between the DR and NODR groups $(t=$ $-1.787, p=0.12)$, the DR and NH groups $(t=2.202, p=0.07$; note that the mean for the DR group was below that for the NH group), or the NODR and NH groups $(t=-0.743, p=0.49)$.

\section{Condition 2 - low-pass cut-off frequency equal to $0.67 f_{\text {edge }}$}

Figure 3 is similar to Fig. 1, but shows results for the low-pass cut-off frequency equal to $0.67 f_{\text {edge }}$ (open symbols). Again, the scores predicted from the SII (filled symbols) were similar for the ears within each triplet, confirming that audibility was well matched across groups. The t-tests showed no significant differences between the DR and NODR groups $(t=$ $-1.663, p=0.15)$, the DR and NH groups $(t=-1.939, p=0.10)$, and the NODR and NH groups $(t=-0.678, p=0.52)$.

Figure 4 is like Fig. 2, but shows SII-relative scores for the low-pass cut-off frequency equal to $0.67 f_{\text {edge }}$ (open symbols). The t-tests showed no significant differences between the DR and NODR groups $(t=-1.513, p=0.18)$, the DR and NH groups $(t=$ $-1.262, p=0.25)$, and the NODR and NH groups $(t=-0.299, p=0.78)$.

Overall, these results do not support the idea that DRs are associated with an enhanced ability to process speech information contained in frequency components below $f_{\text {edge. }}$

\section{Discussion}

The aim of this study was to assess whether children with mid-frequency or high-frequency DRs (the DR group) would demonstrate enhanced abilities to process low-frequency speech information relative to children without DRs (the NODR group) or relative to children with normal hearing (the NH group), under conditions where the audibility of the stimuli was well 
matched across groups. Based on studies performed on adults (Vestergaard, 2003; Moore \& Vinay, 2009), it was hypothesized that when the stimuli included all frequencies up to $f_{\text {edge }}$ (condition 1) the DR group would outperform the NODR group, but when the stimuli included frequencies only up to $0.67 \mathrm{f}_{\text {edge }}$ (condition 2 ) the difference between the DR and NODR groups would be smaller or absent, since the enhancement effects found for adults appears to occur mainly for frequencies just below $f_{\text {edge. }}$. No definite hypothesis was made about the relative performance of the DR group and the NH group in conditions 1 and 2, since any beneficial effects of enhanced central processing for the DR group might be offset by suprathreshold deficits in auditory processing associated with their hearing loss for frequencies below $\mathrm{f}_{\text {edge }}$ (Moore, 2007). In fact, the results provided no evidence of enhanced abilities to process low-frequency speech information for children with DRs (the DR group) compared to children without DRs or to children with normal hearing.

There are several possible reasons for the discrepancy between the results for adults with DR and children with DR. It is possible that extended experience of a basal DR is required for beneficial neural plasticity to occur and this extended experience is lacking in children. This seems unlikely for some of the children in the present study, who were as old as 13 years. Alternatively, the discrepancy between the results for children and adults with DRs could be related to the fact that most of the children in the present study had hearing loss from early on in their life, whereas at least some of the adults tested by Vestergaard (2003) and by Moore and Vinay (2009) had acquired hearing loss. Given that the organization and development of cortical auditory pathways is determined, in part, by input signals, then cortical organization and development must be altered in cases of congenital hearing loss (Sharma et al., 2002; Eggermont \& Ponton, 2003; Sharma et al., 2005; Sharma et al., 2007). All of the hearing-impaired children tested in the present study were regular users of hearing aids from an early age. However, for children with extensive basal DRs, even amplified 
stimuli do not stimulate the central auditory regions tuned to medium and high frequencies. The failure to activate part of the auditory association areas early in life could lead to these areas becoming engaged in processing inputs from other modalities (Lee et al., 2001; Fine et al., 2005; Sharma et al., 2015). This could prevent those areas from becoming involved in the processing of low-frequency acoustic information and hence prevent the "enhancement" in the processing of information from frequencies below $f_{\text {edge. }}$. Further research using imaging and electrophysiological techniques is necessary to establish the extent to which the central auditory pathways are altered in children with extensive DRs that were present early in life.

The absence of significant differences in performance between the DR and NH groups and between the NODR and NH groups is consistent with a previous study comparing the perception of low-pass filtered speech for children with moderate-to-severe hearing loss and children with normal hearing (Zupan \& Dempsey, 2013). However, in that study no test for DRs was performed, so it cannot be determined if there were any effects of DRs.

The present study had several limitations. First, the number of children tested was small, so statistical power was limited. However, the results did not even show a trend in the hypothesized direction. Second, speech perception was assessed using VCV stimuli. Tests using other speech materials, or psychophysical measures of frequency discrimination or amplitude-modulation detection, might reveal differences in performance between hearingimpaired children with and without DRs. Third, the value of $f_{\text {edge }}$ cannot be determined as precisely for children as it can for adults. Fourth, the children in the DR and NODR groups were not perfectly matched in terms of low-frequency hearing. However, the use of frequency-specific amplification prescribed using DSL 4.1 did ensure similar audibility for all children within each triplet. Fifth, three of the ears in the DR group had only a restricted mid-frequency DR, extending up to $2 \mathrm{kHz}$, with recovery of function at 3 and $4 \mathrm{kHz}$. This might have limited the extent of any cortical re-organization. Finally, triplet 5 and triplet 6 
each contained hearing-impaired ears with and without DRs from the same child. This complicates the interpretation of the results.

The perceptual effects of unilateral DRs have not been studied extensively. Heggdal et al. (2013) assessed the auditory processing of stimuli with frequencies below $\mathrm{f}_{\text {edge }}$ for adult participants with unilateral DRs. They also tested participants without DRs matched for the amount of hearing loss. Heggdal et al. (2013) used a frequency discrimination task, with center frequencies of 0.5 and $0.25 \mathrm{kHz}$. The former was closest to the values of $\mathrm{f}_{\text {edge }}$ estimated using the TEN(HL) test (Moore, 2004). They found that ears with DRs performed best overall, indicating a perceptual enhancement effect. Ears with a contralateral DR performed significantly better than ears without a contralateral DR at $0.5 \mathrm{kHz}$, while the opposite was true at $0.25 \mathrm{kHz}$. Thus, it appears that for adults there are ear-specific effects of DRs, but with contralateral influences. In our data, the results for the two children with unilateral DRs did not differ in any systematic way from those for the other triplets. For both triplets 5 and 6, the measured speech scores for the hearing-impaired children were close to those predicted from the SII for both ears. In other words, the presence of a DR in one ear did not influence the outcome. However, further research is needed to clarify the changes in auditory processing produced by unilateral DRs in both children and adults.

\section{Summary and Conclusions}

The ability to identify low-pass filtered VCV stimuli was compared for three groups: hearing impaired with DRs with edge frequency $\mathrm{f}_{\text {edge }}$ (group DR), hearing impaired without DRs (group NODR), and normal hearing (group NH). Groups DR and NODR were matched in their audiometric thresholds below $\mathrm{f}_{\text {edge }}$ and in age, and the audibility of the stimuli was well matched across all three groups by the use of frequency-selective amplification for the two groups with hearing impairment, as indicated by values of the SII. Group DR did not show enhanced speech identification relative to either of the other two groups. Rather, performance 
was similar across groups or was slightly but non-significantly poorer for group DR. The results differ from those found for adults, perhaps because the early onset of hearing loss for the children with DRs tested here led to changes in responsiveness of secondary auditory cortical areas that would normally be tuned to frequencies within the DRs. Those areas may instead have become responsive to other modalities such as vision, preventing the areas from contributing to the analysis of auditory information from frequencies below $\mathrm{f}_{\text {edge. }}$.

\section{References}

ANSI 1997. ANSI S3.5-1997, Methods for the calculation of the speech intelligibility index. New York: American National Standards Institute.

Cohen L.G., Celnik P., Pascual-Leone A., Corwell B., Falz L., et al. 1997. Functional relevance of cross-modal plasticity in blind humans. Nature, 389, 180-183.

Dong S., Rodger J., Mulders W.H.A.M. \& Robertson D. 2010. Tonotopic changes in GABA receptor expression in guinea pig inferior colliculus after partial unilateral hearing loss. Brain Res, 1342, 24-32.

Eggermont J.J. 2017. Acquired hearing loss and brain plasticity. Hear Res, 343, 176-190.

Eggermont J.J. \& Ponton C.W. 2003. Auditory-evoked potential studies of cortical maturation in normal hearing and implanted children: correlations with changes in structure and speech perception. Acta Otolaryngol, 123, 249-252.

Fine I., Finney E.M., Boynton G.M. \& Dobkins K.R. 2005. Comparing the effects of auditory deprivation and sign language within the auditory and visual cortex. $J$ Cogn Neurosci, 17, 1621-1637.

Harrison R.V. 2001. Age-related tonotopic map plasticity in the central auditory pathways. Scand Audiol Suppl, 30, 8-14.

Heggdal P.O., Lind O. \& Brannstrom K.J. 2013. Frequency discrimination in ears with and without contralateral cochlear dead regions. Int J Audiol, 52, 553-557.

Irvine D.R. \& Wright B.A. 2005. Plasticity of spectral processing. Int Rev Neurobiol, 70, 435472.

Kluk K. \& Moore B.C.J. 2006. Dead regions in the cochlea and enhancement of frequency discrimination: Effects of audiogram slope, unilateral versus bilateral loss, and hearing-aid use. Hear Res, 222, 1-15.

Kral A., Hartmann R., Tillein J., Heid S. \& Klinke R. 2001. Delayed maturation and sensitive periods in the auditory cortex. Audiol Neurootol, 6, 346-362.

Lee D.S., Lee J.S., Oh S.H., Kim S.K., Kim J.W., et al. 2001. Cross-modal plasticity and cochlear implants. Nature, 409, 149-150.

Malicka A.N., Munro K.J., Baer T., Baker R.J. \& Moore B.C.J. 2013. The effect of low-pass filtering on identification of nonsense syllables in quiet by school-age children with and without cochlear dead regions. Ear Hear, 34, 458-469.

Malicka A.N., Munro K.J. \& Baker R.J. 2009. Fast method for psychophysical tuning curve measurement in school-age children. Int J Audiol, 48, 546-553. 
Moore B.C.J. 2001. Dead regions in the cochlea: diagnosis, perceptual consequences, and implications for the fitting of hearing aids. Trends Amplif, 5, 1-34.

Moore B.C.J. 2004. Dead regions in the cochlea: conceptual foundations, diagnosis, and clinical applications. Ear Hear, 25, 98-116.

Moore B.C.J. 2007. Cochlear hearing loss- physiological, psychological and technical issues. Chichester: England: Wiley.

Moore B.C.J., Glasberg B.R. \& Stone M.A. 2004. New version of the TEN test with calibrations in dB HL. Ear Hear, 25, 478-487.

Moore B.C.J. \& Malicka A.N. 2013. Cochlear dead regions in adults and children: diagnosis and clinical implications. Semin Hear, 34, 037-050.

Moore B.C.J. \& Vinay S.N. 2009. Enhanced discrimination of low- frequency sounds for subjects with high- frequency dead regions. Brain, 132, 524-536.

Nudo R.J. 2006. Plasticity. NeuroRx, 3, 420-427.

Robertson D. \& Irvine D.R. 1989. Plasticity of frequency organization in auditory cortex of guinea pigs with partial unilateral deafness. J Comp Neurol, 282, 456-471.

Seewald R., Moodie S., Scollie S. \& Bagatto M. 2005. The DSL method for pediatric hearing instrument fitting: historical perspective and current issues. Trends Amplif, 9, 145157.

Sek A., Alcantara J., Moore B.C.J., Kluk K. \& Wicher A. 2005. Development of a fast method for determining psychophysical tuning curves. Int J Audiol, 44, 408-420.

Sharma A., Campbell J. \& Cardon G. 2015. Developmental and cross-modal plasticity in deafness: evidence from the P1 and N1 event related potentials in cochlear implanted children. Int J Psychophysiol, 95, 135-144.

Sharma A., Dorman M.F. \& Kral A. 2005. The influence of a sensitive period on central auditory development in children with unilateral and bilateral cochlear implants. Hear Res, 203, 134-143.

Sharma A., Dorman M.F. \& Spahr A.J. 2002. A sensitive period for the development of the central auditory system in children with cochlear implants: implications for age of implantation. Ear Hear, 23, 532-539.

Sharma A., Gilley P.M., Dorman M.F. \& Baldwin R. 2007. Deprivation-induced cortical reorganization in children with cochlear implants. Int J Audiol, 46, 494-499.

Thai-Van H., Micheyl C., Norena A., Veuillet E., Gabriel D., et al. 2007. Enhanced frequency discrimination in hearing-impaired individuals: a review of perceptual correlates of central neural plasticity induced by cochlear damage. Hear Res, 233, 14-22.

Vestergaard M.D. 2003. Dead regions in the cochlea: implications for speech recognition and applicability of articulation index theory. Int J Audiol, 42, 249-261.

Zupan B. \& Dempsey L. 2013. Narrative comprehension abilities of children with typical hearing and children using hearing aids: A pilot study. Volta Rev, 113, 5-27. 
Table 1. For each ear in each triplet, the table shows sex, age (years), test ear, and audiometric thresholds in $\mathrm{dB}$ HL. $\mathrm{DR}=$ with dead region; NODR = hearing-impaired without dead region; $\mathrm{NH}=$ normal hearing. For the $\mathrm{NH}$ group, all thresholds were $\leq 20 \mathrm{~dB}$ HL. For the ears of the hearing-impaired children, a participant identifier is given as $\mathrm{P}$ followed by a number. This allows identification of cases where both ears were tested. For example, the right and left ears of $\mathrm{P} 1$ were in triplets 1 and 2, respectively. For triplet 5 and triplet 6 , the DR and NODR ears were from the same child (P6 and P7, respectively). For the DR participants in triplets 3, 4 and 6, the DRs were not continuous. The * symbols indicate frequencies falling within a DR. The lowest frequency at which a DR was present was taken as the value of $f_{\text {edge. }}$.

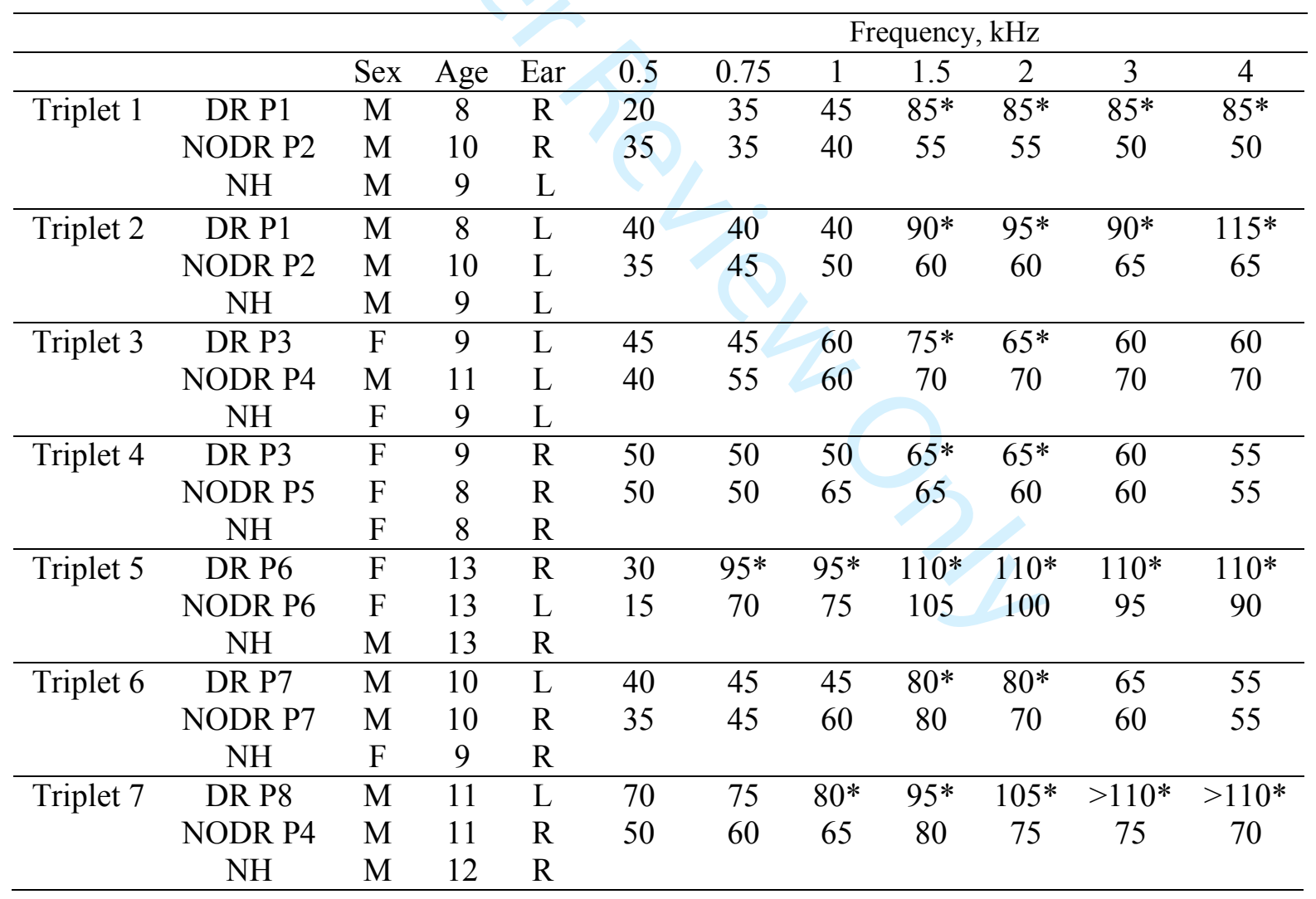


Figures

Figure 1. Open symbols show mean VCV scores for condition 1 (low-pass filtering at $f_{\text {edge }}$ ) for each ear in each triplet. Within each triplet there was one ear with a DR (DR), one ear with no DR (NODR), and one ear with normal hearing (NH). The filled symbols show scores predicted from the SII.

Figure 2. Obtained VCV scores for condition 1 plotted relative to scores predicted from the SII. Otherwise, as Figure 1.

Figure 3. As Figure 1, but for condition 2 (low-pass filtering at $0.67 \mathrm{f}_{\text {edge }}$ ).

Figure 4. As Figure 2, but for condition 2. 


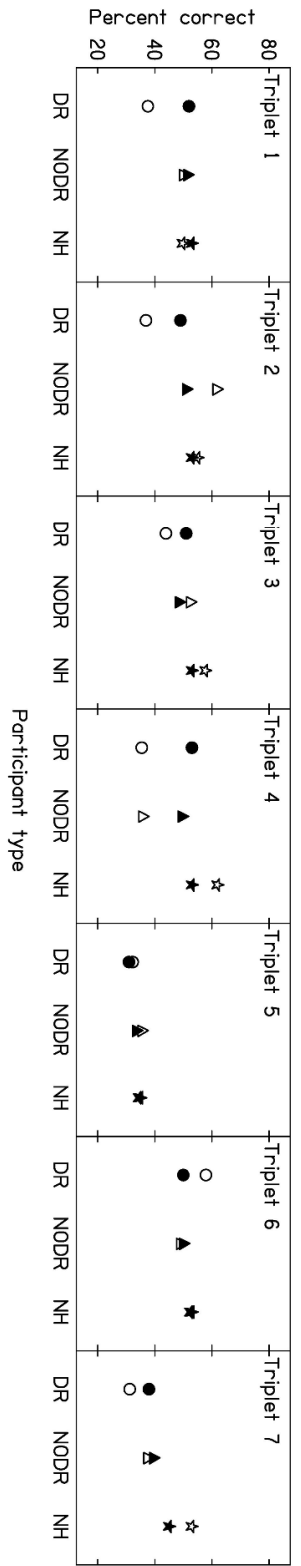

Figure 1. Open symbols show mean VCV scores for condition 1 (low-pass filtering at fedge) for each ear in each triplet. Within each triplet there was one ear with a DR (DR), one ear with no DR (NODR), and one ear with normal hearing $(\mathrm{NH})$. The filled symbols show scores predicted from the SII.

$$
220 \times 1191 \mathrm{~mm}(300 \times 300 \mathrm{DPI})
$$




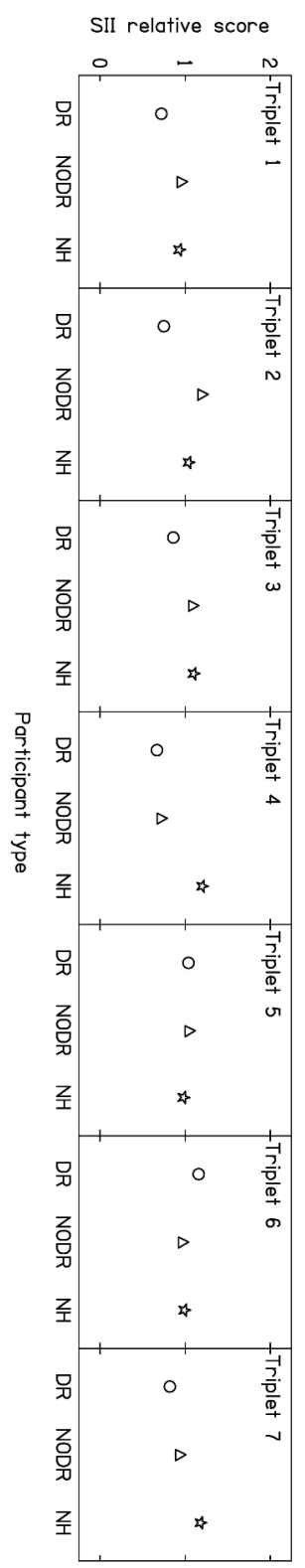

Figure 2. Obtained VCV scores for condition 1 plotted relative to scores predicted from the SII. Otherwise, as Figure 1.

$221 \times 1194 \mathrm{~mm}(300 \times 300 \mathrm{DPI})$ 


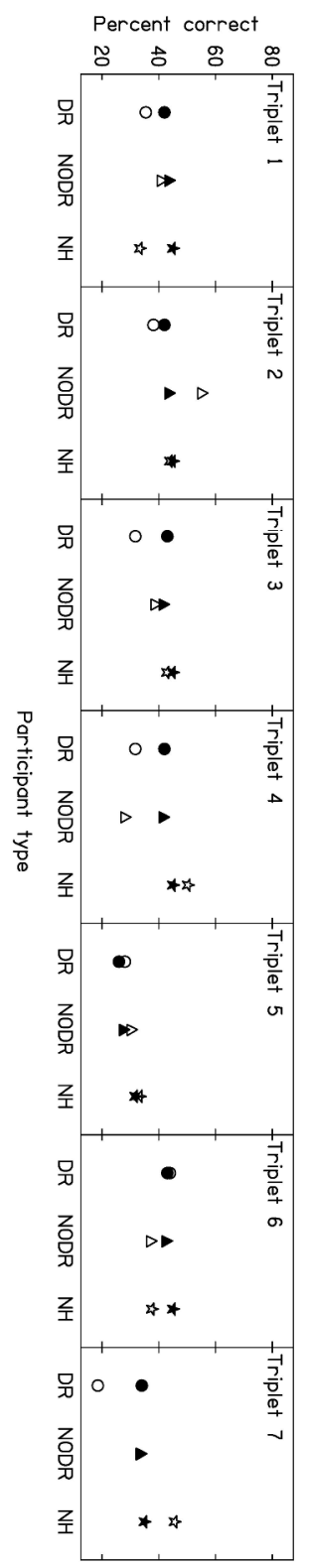

Figure 3. As Figure 1, but for condition 2 (low-pass filtering at 0.67 fedge). $220 \times 1191 \mathrm{~mm}(300 \times 300$ DPI $)$ 


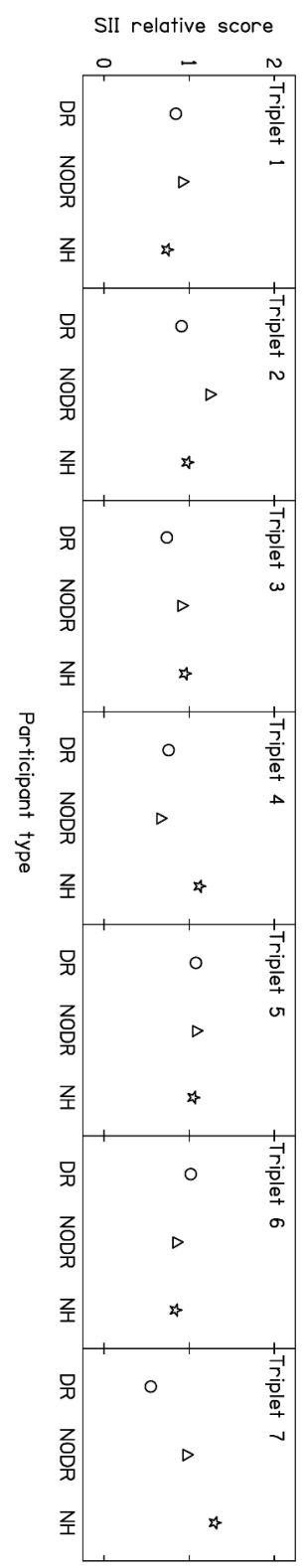

Figure 4. As Figure 2, but for condition 2. $221 \times 1194 \mathrm{~mm}(300 \times 300 \mathrm{DPI})$ 